\title{
Association of NT-proBNP and interleukin-17 levels with heart failure in elderly patients
}

\author{
X.F. Li' ${ }^{1}$, D. Pan ${ }^{2}$, W.L. Zhang ${ }^{3}$, J. Zhou ${ }^{4}$ and J.J. Liang \\ ${ }^{1}$ Department of Cardiology, Liaocheng People's Hospital, Liaocheng, \\ Shandong, China \\ ${ }^{2}$ Department of Cardiology, Liaocheng Maternal and Child Health Hospital, \\ Liaocheng, Shandong, China \\ ${ }^{3}$ Department of Cardiology, The Third People's Hospital of Liaocheng, \\ Shandong, China \\ ${ }^{4}$ Liaocheng Vocational \& Technical College, Liaocheng, Shandong, China \\ ${ }^{5}$ Department of Cardiology, Shandong Provincial Qianfoshan Hospital, Jinan, \\ Shandong, China \\ Corresponding author: J.J. Liang \\ E-mail: liangjiangjiu_1@163.com \\ Genet. Mol. Res. 15 (2): gmr. 15028014 \\ Received November 6, 2015 \\ Accepted January 22, 2016 \\ Published May 13, 2016 \\ DOI http://dx.doi.org/10.4238/gmr.15028014
}

\begin{abstract}
Pro-B-type natriuretic peptide (NT-proBNP) and interleukin-17 (IL-17) are involved in the pathophysiological processes of heart failure; however, the exact role of IL-17 is not clear. We explored the relationship between IL-17 and NT-proBNP, as a clinical parameter, in heart failure. The whole blood IL-17 and NT-proBNP levels and the readmission rates in 70 patients with chronic heart failure class III or IV according to the New York Heart Association and 35 patients with normal heart function (control group) were measured and compared. The left ventricle ejection fractions (LVEFs) and NT-proBNP and IL17 levels in cardiac functional class III $(40.38 \pm 4.76 \%, 7780 \pm 6393$ $\mathrm{pg} / \mathrm{mL}, 8.65 \pm 3.05 \mathrm{pg} / \mathrm{mL}$, respectively) and class IV (31.59 $\pm 4.31 \%$, $13,704 \pm 10,945,21.10 \pm 10.60 \mathrm{pg} / \mathrm{mL}$, respectively) were higher than those in the control group $(61.27 \pm 5.66 \%, 420 \pm 256 \mathrm{pg} / \mathrm{mL}, 3.53 \pm 2.05$
\end{abstract}


$\mathrm{pg} / \mathrm{mL}$, respectively). Compared to the cardiac functional class IV, class III showed significantly higher values for LVEF and NT-proBNP and IL-17 levels $(\mathrm{P}<0.05)$. The readmission rates of the patients in cardiac functional class III at 3 and 6 months (15.7 and 34.4\%, respectively) and cardiac functional class IV at 3 and 6 months (39.5 and 76.3\%, respectively) were significantly higher than those in the control group ( 0 and $5.7 \%$, respectively) $(\mathrm{P}<0.05)$. The NT-proBNP and IL-17 levels increased as the heart function worsened. NT-proBNP and IL-17 may play essential roles in the process of heart failure.

Key words: Congestive heart failure; Interleukin-17; $\mathrm{N}$-terminal pro-brain natriuretic peptide

\section{INTRODUCTION}

Heart failure is a global term for the physiological state in which cardiac output is insufficient in meeting the needs of the body and lungs. Heart failure is a common, costly, disabling, and potentially deadly condition (Flavell and Stevenson, 2001). Management of heart failure has been challenging in clinical medicine. It is clear to us that N-terminal probrain natriuretic peptide (NT-proBNP) plays a role in the occurrence and progression of heart failure (Lainchbury et al., 2009). NT-proBNP has been widely used as a reliable indicator when evaluating the severity and prognosis of the heart failure patients (Galinier et al., 2013; Luers et al., 2013). Recently, much attention has been paid to the role of the cytokines in the pathogenesis of heart failure. Interlukin-17 (IL-17) is an important cytokine to evaluate the severity and prognosis of heart failure (Milovanovic et al., 2012). Both NT-proBNP and IL-17 are involved in the pathophysiological processes of heart failure; however, the exact functional role of IL-17 in the development of heart failure is not clear. We studied and measured the changes in serum levels of NT-proBNP and IL-17 in senior heart failure patients for further understanding of their roles in the pathogenesis of heart failure.

\section{MATERIAL AND METHODS}

\section{Patients}

All patients were hospitalized in the People's Hospital of Liaocheng City from January 2011 to August 2012. All patients were diagnosed with heart failure based on medical history, physical exam, electrocardiogram (EKG), chest X-ray, and echocardiogram. In our study, heart failure is defined as systolic dysfunction if the ejection fraction from echocardiogram was less than $45 \%$. We selected 70 patients with heart failure. Based on New York Heart Association (NYHA) heart failure classification, 32 of them were class III and 38 were class IV. In the 70 heart failure patients, there were 40 male and 30 female. Average age of the patients was $73.54 \pm 11.5$ years old. The etiology included ischemic cardiomyopathy, dilated cardiomyopathy, and hypertensive heart disease. The control group included 35 patients with normal cardiac function (19 male and 16 female, average age $70.75 \pm 14.08$ years old). There were no significant differences in age and sex between the study group and control group.

Exclusion criteria included: 1) chronic or acute infection; 2) autoimmune disease; 
3) unstable angina and acute cardiac infarction within 3 months; 4) active rheumatoid disease within 3 months; 5) congenital heart disease; 6) diabetes mellitus, hyperthyroidism, and other endocrinology disorders; 7) recent use of immunosuppressive medications (e.g. corticosteroids); 8) abnormal hepatic or renal function; 9) cancer; or 10) pregnancy. Data collected included demographic information (age, gender, ethnic background, occupation, diet, etc.), medical history, physical exam, laboratory tests, EKG, chest X-ray, echocardiogram, and medication use.

\section{Blood sample collection}

Venous blood samples were collected at 6 am after fasting. EDTA (Beijing Solarbio Science and Technology Co., Ltd., Beijing, China) was added to samples to prevent coagulation. The samples were then processed for serum levels of NT-proBNP and IL-17.

\section{Detection of NT-proBNP and IL-17}

NT-proBNP was measured with AQT90 FLEX immunity analyzer and relative agents (Radiometer Medical APS, Denmark). IL-17 was measured with enzyme-linked immunosorbent assay (ELISA) kit (4A Biotech Co., Ltd., Beijing, China) and enzyme mark instrument (PerkinElmer Inc., USA).

\section{Cardiac structure and left ventricle ejection fraction (LVEF)}

The cardiac structure and LVEF were evaluated with a Nemio 30 color ultrasound machine (Toshiba, Shanghai, China) by a physician sonographer.

\section{Statistical analysis}

The measured data are reported as means \pm standard deviation and analyzed by the SPSS13.0 software (Chicago, IL, USA). The comparison between the two groups was analyzed with the Student $t$-test, and multiple group comparisons were analyzed with ANOVA. Pairwise comparisons were performed with Bonferroni and least significant difference methods. Counted data were compared using chi-square test, and correlations were analyzed by linear correlation analysis. The difference was considered statistically significant when $\mathrm{P}<0.05$.

\section{RESULTS}

\section{Comparison of LVEF}

As shown in Table 1, the LVEFs of the patients in cardiac functional class III $(40.38 \pm 4.76 \%)$ and IV $(31.59 \pm 4.31 \%)$ were lower than those in the control group $(61.27$ $\pm 5.66 \%$ ), while the LVEF in cardiac functional class III was significantly higher than that in class IV $(\mathrm{P}<0.05)$. 
Table 1. Comparison of LVEF among different groups of heart function.

\begin{tabular}{l|c|c}
\hline Groups & Cases & LVEF (\%) \\
\hline Control & 35 & $61.27 \pm 5.66$ \\
\hline Cardiac functional class III (NYHA) & 32 & $40.38 \pm 4.76^{\Delta}$ \\
\hline Cardiac functional class IV (NYHA) & 38 & $31.59 \pm 4.31^{\Delta}$ \\
\hline
\end{tabular}

${ }_{\triangle} \mathrm{P}<0.05$ compared to the control group; ${ }^{-\mathrm{P}}<0.05$ compared to cardiac functional class III.

\section{Comparison of NT-proBNP and IL-17}

The levels of NT-proBNP and IL-17 elevated as cardiac function worsened and positively correlated with the degree of heart failure (Table 2). NT-proBNP levels in cardiac functional class III $(7780 \pm 6393 \mathrm{pg} / \mathrm{mL})$ and IV $(13,704 \pm 10,945 \mathrm{pg} / \mathrm{mL})$ were higher than those in the control group $(420 \pm 256 \mathrm{pg} / \mathrm{mL})$, while the NT-proBNP levels in cardiac functional class III were significantly higher than those in cardiac functional class IV (P $<$ $0.05)$. Similarly, IL-17 levels in cardiac functional class III $(8.65 \pm 3.05 \mathrm{pg} / \mathrm{mL})$ and IV $(21.10$ $\pm 10.60 \mathrm{pg} / \mathrm{mL})$ were higher than those in the control group $(3.53 \pm 2.05)$, while the IL-17 levels in cardiac functional class III were significantly higher than those in cardiac functional class IV $(\mathrm{P}<0.05)$.

Table 2. Comparison of NT-proBNP and IL-17 among different groups of heart function.

\begin{tabular}{l|c|c|c}
\hline Groups & Cases & NT-proBNP $(\mathrm{pg} / \mathrm{mL})$ & $\mathrm{IL}-17(\mathrm{pg} / \mathrm{mL})$ \\
\hline Control & 35 & $420 \pm 256$ & $3.53 \pm 2.05$ \\
\hline Cardiac functional class III (NYHA) & 32 & $7,780 \pm 6,393^{\mathbf{4}}$ & $8.65 \pm 3.05^{\mathbf{\Lambda}}$ \\
\hline Cardiac functional class IV (NYHA) & 38 & $13,704 \pm 10,945^{\mathbf{\bullet}}$ & $21.10 \pm 10.60^{\mathbf{\bullet}}$ \\
\hline
\end{tabular}

$\Delta \mathrm{P}<0.05$ compared to the control group, $\mathbf{}^{\mathrm{P}}<0.05$ compared to cardiac functional class III.

\section{Readmission rate}

The readmission rates of the patients in cardiac functional class III at 3 months $(15.7 \%$; $\mathrm{N}=5)$ and 6 months $(34.4 \% ; \mathrm{N}=11)$ were significantly higher than those in the control group (3 months $=0 ; 6$ months $=5.7 \% ; \mathrm{N}=2$ ) (Table 3; $\mathrm{P}<0.05)$. The readmission rates of the patients in cardiac functional class IV at 3 months $(39.5 \% ; \mathrm{N}=15)$ and 6 months $(76.3 \% ; \mathrm{N}=29)$ were significantly higher than those in the control group ( 3 months $=0 ; 6$ months $=5.7 \% ; \mathrm{N}=2)(\mathrm{P}<$ $0.05)$. The readmission rates positively correlated with the NT-proBNP and IL-17 levels $(\mathrm{P}<0.05)$.

Table 3. Comparison of the readmission rate among different groups of heart function.

\begin{tabular}{l|c|c|c|c|c}
\hline Groups & Cases & NT-proBNP $(\mathrm{pg} / \mathrm{mL})$ & IL-17 (pg/mL) & $\begin{array}{c}\text { Readmission rate at 3 } \\
\text { months [\% (N)] }\end{array}$ & $\begin{array}{c}\text { Readmission rate at 6 } \\
\text { months [\% (N)] }\end{array}$ \\
\hline Control & 35 & $420 \pm 256$ & $3.53 \pm 2.05$ & 0 & $5.7 \%(2)$ \\
\hline Cardiac functional class III (NYHA) & 32 & $7,780 \pm 6,393$ & $8.65 \pm 3.05$ & $15.7 \%(5)^{\mathbf{\Lambda}}$ & $34.4 \%(11)^{\mathbf{\Lambda}}$ \\
\hline Cardiac functional class IV (NYHA) & 38 & $13,704 \pm 10,945$ & $21.1 \pm 10.60$ & $39.5 \%(15)^{\mathbf{\Lambda}}$ & $76.3 \%(29)^{\mathbf{\Lambda}}$ \\
\hline
\end{tabular}

${ }^{\Delta} \mathrm{P}<0.05$ compared to the control group, ${ }^{\square} \mathrm{P}<0.05$ compared to cardiac functional class III.

\section{DISCUSSION}

Heart failure is the end stage of all cardiac diseases and it is one of the leading causes of death and disability in humans. During the pathophysiological changes of chronic heart 
failure, the excessive activation of nerve roots and humoral factors, such as the sympathetic nervous system, renin-angiotensin-aldosterone system (RAAS), BNP, antidiuretic hormone $(\mathrm{ADH})$, and endothelin (ET), constitute an important mechanism in the occurrence and development of heart failure (Mann and Young, 1994; Sanghi et al., 2005; Palazzuoli et al., 2012; Lang and Struthers, 2013; Scollan et al., 2013; Skiendzielewski and Werner, 2014). In recent years, BNP has been seen as a natural antagonist in the RASS. Substantial evidence indicates that serum BNP levels are elevated with worsening heart failure (Niu et al., 2014). BNP plays a role as an antagonist in the RASS and the sympathetic nervous system (SNS) in both peripheral and central nervous systems to maintain fluid and electrolyte balance (Cameron and Ellmers, 2003). In addition, BNP, as an anti-fibrosis factor from the cardiac ventricle, can inhibit the proliferation of vascular smooth muscle cells, mesangial cells, and fibroblasts. Thus, BNP can regulate ventricular remodeling locally. NT-proBNP is the amino-terminal fragment of the B-type natriuretic peptide prohormone. Compared with BNP, NT-proBNP is an inactive aminoterminal fragment with a long half-life (60-120 $\mathrm{min})$. It is stable, sensitive, and reproducible, and it has become an important indicator in the clinical diagnosis of heart failure.

IL-17 has been recently identified as a cellular inflammatory cytokine. IL-17 can inhibit the reconstruction of the heart by myocardial fibrosis through dissolution, breakage, and reduced synthesis of intercellular collagen by activation of the matrix metalloproteinases (MMPs). Previous studies showed that lower serum IL-17 levels helped to improve cardiac function in heart failure patients. Many scholars believe that the IL-17 plays a role through two pathways: the nuclear factor (NF)- $\mathrm{BB}-\mathrm{DNA}$ pathway and mitogen-activated protein (MAP) kinase pathway. In addition, there could be a role of IL-17 through the tumor necrosis factor

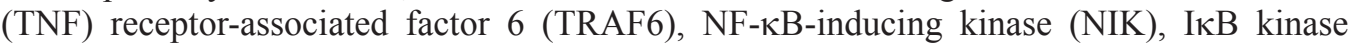
(IKK)- $\alpha$, or JAK-STAT pathways (Schwandner et al., 2000; Wuyts et al., 2005)

Research using animal models showed that IL-17 caused damage to the heart through the following mechanisms: 1) IL-17 can exert direct toxic effects on myocardial cells; 2) IL-17 enhances nitric oxide synthase (iNOS) activity, which causes increased release of NO by the vascular endothelium and damage to the vascular endothelium (Krstić et al., 2013); 3) IL-17 reduces myocardial intracellular calcium levels by activating the nerve myelin sheath esterase and triggering a series of inflammatory reactions (Hu et al., 2014); and 4) IL-17 can induce the expression, increase the secretion, and enhance the activity of inflammatory factors such as IL-6 and IL-1 $\beta$ (Lee et al., 2009). This eventually leads to cardiac hypertrophy, vascular endothelial damage, increased cellular necrosis, accelerated myocardial apoptosis, and extracellular matrix remodeling, thus accelerating the process of heart failure (Eid et al., 2009).

It has been reported that in chronic heart failure, there was no significant increase in IL-17 levels in class I and II heart failure but there was a significant increase in IL-17 levels in class III and IV heart failure. This is consistent with the findings in our present study. Heart failure contributes to the production of TNF- $\alpha$ and IL-17 (and vice versa), which constitutes a vicious cycle of cardiac remodeling. Studies have shown that there is a change in the signal amplification cascade of IL-17/gp130-JAK-STAT in patients with end-stage heart failure (Podewski et al., 2003). After ischemia reperfusion, injection of IL-17/soluble IL-17R complex prevents myocardial apoptosis and reduces the size of the myocardial infarct. This indicates the protective effect of IL-17 (Matsushita et al., 2005).

Our study is consistent with the reports of Li et al. (2010), which show that the ratio of Th17/CD4 + T cells in peripheral blood was significantly higher in patients with chronic heart failure class III and IV as compared to the patients with chronic heart failure class I and II. In 
our study, there was a parallel increase in the levels of NT-proBNP and IL-17 in patients with heart failure, which may indicate that both are involved in the development of heart failure. Furthermore, the levels of both factors increased as the degree of heart failure increased. BNP has become a reliable indicator to assess the prognosis and degree of heart failure and IL-17 can be used to assess the prognosis and degree of heart failure as well. Further studies with expanded sample size are needed to address the specific relationship between NT-proBNP and IL-17 and the exact mechanism underlying heart failure involving serum IL-17 levels.

\section{Conflicts of interest}

The authors declare no conflict of interest.

\section{ACKNOWLEDGMENTS}

Research supported by the Shandong Provincial Natural Science Foundation, China (\#ZR2010HM116).

\section{REFERENCES}

Cameron VA and Ellmers LJ (2003). Minireview: natriuretic peptides during development of the fetal heart and circulation. Endocrinology 144: 2191-2194. http://dx.doi.org/10.1210/en.2003-0127

Eid RE, Rao DA, Zhou J, Lo SF, et al. (2009). Interleukin-17 and interferon-gamma are produced concomitantly by human coronary artery-infiltrating $\mathrm{T}$ cells and act synergistically on vascular smooth muscle cells. Circulation 119: 14241432. http://dx.doi.org/10.1161/CIRCULATIONAHA.108.827618

Flavell C and Stevenson LW (2001). Take heart with heart failure. Circulation 104: e89-e91. http://dx.doi.org/10.1161/ $\underline{\text { hc4301.099136 }}$

Galinier M, Berry M, Delmas C and Fournier P (2013). [Interest of NT-proBNP in chronic heart failure follow-up]. Ann. Biol. Clin. (Paris) 71: 39-45.

Hu MH, Zheng QF, Jia XZ, Li Y, et al. (2014). Neuroprotection effect of interleukin (IL)-17 secreted by reactive astrocytes is emerged from a high-level IL-17-containing environment during acute neuroinflammation. Clin. Exp. Immunol. 175: 268-284.http://dx.doi.org/10.1111/cei.12219

Krstić J, Jauković A, Mojsilović S, Đorđević IO, et al. (2013). In vitro effects of IL-17 on angiogenic properties of endothelial cells in relation to oxygen levels. Cell Biol. Int. 37: 1162-1170.

Lainchbury JG, Troughton RW, Strangman KM, Frampton CM, et al. (2009). N-terminal pro-B-type natriuretic peptideguided treatment for chronic heart failure: results from the BATTLESCARRED (NT-proBNP-Assisted Treatment To Lessen Serial Cardiac Readmissions and Death) trial. J. Am. Coll. Cardiol. 55: 53-60. http://dx.doi.org/10.1016/j. jacc.2009.02.095

Lang CC and Struthers AD (2013). Targeting the renin-angiotensin-aldosterone system in heart failure. Nat. Rev. Cardiol. 10: 125-134. http://dx.doi.org/10.1038/nrcardio.2012.196

Lee JH, Cho ML, Kim JI, Moon YM, et al. (2009). Interleukin 17 (IL-17) increases the expression of Toll-like receptor-2, 4, and 9 by increasing IL-1beta and IL-6 production in autoimmune arthritis. J. Rheumatol. 36: 684-692. http:// dx.doi.org/10.3899/jrheum.080169

Li N, Bian HJ, Zhang J and Ji XP (2010). Detection and significance of Th17 and Treg cells in patients with chronic heart failure. J. Shandong Univ. 411: 1963-1968.

Luers C, Sutcliffe A, Binder L, Irle S, et al. (2013). NT-proANP and NT-proBNP as prognostic markers in patients with acute decompensated heart failure of different etiologies. Clin. Biochem. 46: 1013-1019. http://dx.doi.org/10.1016/j. clinbiochem.2013.03.014

Mann DL and Young JB (1994). Basic mechanisms in congestive heart failure. Recognizing the role of proinflammatory cytokines. Chest 105: 897-904. http://dx.doi.org/10.1378/chest.105.3.897

Matsushita K, Iwanaga S, Oda T, Kimura K, et al. (2005). Interleukin-6/soluble interleukin-6 receptor complex reduces infarct size via inhibiting myocardial apoptosis. Lab. Invest. 85: 1210-1223. http://dx.doi.org/10.1038/ labinvest. 3700322 
Milovanovic M, Pesic G, Nikolic V, Jevtovic-Stoimenov T, et al. (2012). Vitamin D deficiency is associated with increased IL-17 and TNFa levels in patients with chronic heart failure. Arq. Bras. Cardiol. 98: 259-265. http://dx.doi. org $10.1590 / \mathrm{S} 0066-782 X 2012005000019$

Niu JM, Ma ZL, Xie C and Zhang ZQ (2014). Association of plasma B-type natriuretic peptide concentration with myocardial infarct size in patients with acute myocardial infarction. Genet. Mol. Res. 13: 6177-6183. http://dx.doi. org/10.4238/2014.February.21.6

Palazzuoli A, Caputo M, Calabrò A and Nuti R (2012). Clinical impact of BNP and other emerging biomarkers in heart failure evaluation and management. Minerva Cardioangiol. 60: 183-194.

Podewski EK, Hilfiker-Kleiner D, Hilfiker A, Morawietz H, et al. (2003). Alterations in Janus kinase (JAK)-signal transducers and activators of transcription (STAT) signaling in patients with end-stage dilated cardiomyopathy. Circulation 107: 798-802.http://dx.doi.org/10.1161/01.CIR.0000057545.82749.FF

Sanghi P, Uretsky BF and Schwarz ER (2005). Vasopressin antagonism: a future treatment option in heart failure. Eur. Heart J. 26: 538-543. http://dx.doi.org/10.1093/eurheartj/ehi145

Schwandner R, Yamaguchi K and Cao Z (2000). Requirement of tumor necrosis factor receptor-associated factor (TRAF)6 in interleukin 17 signal transduction. J. Exp. Med. 191: 1233-1240. http://dx.doi.org/10.1084/jem.191.7.1233

Scollan KF, Bulmer BJ and Sisson DD (2013). Validation of a commercially available enzyme immunoassay for measurement of plasma antidiuretic hormone concentration in healthy dogs and assessment of plasma antidiuretic hormone concentration in dogs with congestive heart failure. Am. J. Vet. Res. 74: 1206-1211. http://dx.doi. org/10.2460/ajvr.74.9.1206

Skiendzielewski J and Werner B (2014). The relevance of endothelin-1 in evaluation of the stage of heart failure in infants with ventricular septal defect. Kardiol. Pol. 72: 459-464.http://dx.doi.org/10.5603/KP.a2013.0351

Wuyts WA, Vanaudenaerde BM, Dupont LJ, Van Raemdonck DE, et al. (2005). Interleukin-17--induced interleukin-8 release in human airway smooth muscle cells: role for mitogen-activated kinases and nuclear factor-kappaB. J. Heart Lung Transplant. 24: 875-881. http://dx.doi.org/10.1016/j.healun.2004.05.003

Zhu SJ and Wang J (2001). Energy metabolic therapy can be used as a new target for treatment of heart failure. South China J. Crdiovascular Dis 2010: 97-98. 